\title{
Correction to: A Fuzzy Ontology Based Approach to Support Product Eco-Ensign
}

Chaimae Abadi D, Imad Manssouri, and Asmae Abadi

\section{Correction to:}

Chapter "A Fuzzy Ontology Based Approach to Support

Product Eco-Design"' in: T. Masrour et al. (eds.),

Artificial Intelligence and Industrial Applications, Advances

in Intelligent Systems and Computing 1193, https://doi.org/10.1007/978-3-030-51186-9_1

In the original version of the book, the co-author name "Manssour" has been changed to "Manssouri" in the chapter "A Fuzzy Ontology Based Approach to Support Product Eco-Design". The erratum chapter and the book have been updated with the change. 